\title{
ERRATA FOR SECTION 1.10.7
}

(1) Due to a coding mistake the algorithm used to solve the test problems in Sections 1.10.7.2 and 1.10.7.3 of Part I (see Acta Numerica 1994) was not a genuine conjugate gradient algorithm. Indeed, the search direction sequence $\left\{w^{n}\right\}_{n \geq 0}$ was improperly defined leading to a slow convergence; for the 'small' values of $k$ (e.g. $10^{2}, 10^{3}$ ) the computed results were essentially correct, but for larger values the slow convergence prevented us from reaching the correct limit since we stopped iterating after a fixed number of iterations ( 300 or 500 , depending of the test problem).

The results obtained with the corrected algorithm are given in Tables 1 to 5 (which replace the coresponding tables of Part I, on pages 345, 356, 357 and 364). Due to the fast convergence properties we have been able to consider much larger values of the penalty parameter $k$ than in Part I (upto $10^{10}$ ). The comments (i) and (ii) in pages 345 and 356 of Part I are still relevant for the values of $k$ used here since the convergence is achieved for a number of iterations which is much smaller than the dimension of the

Table 1. Summary of numerical results (target defined by (1.461)); $T=3$, $h=\Delta t=10^{-2}$.

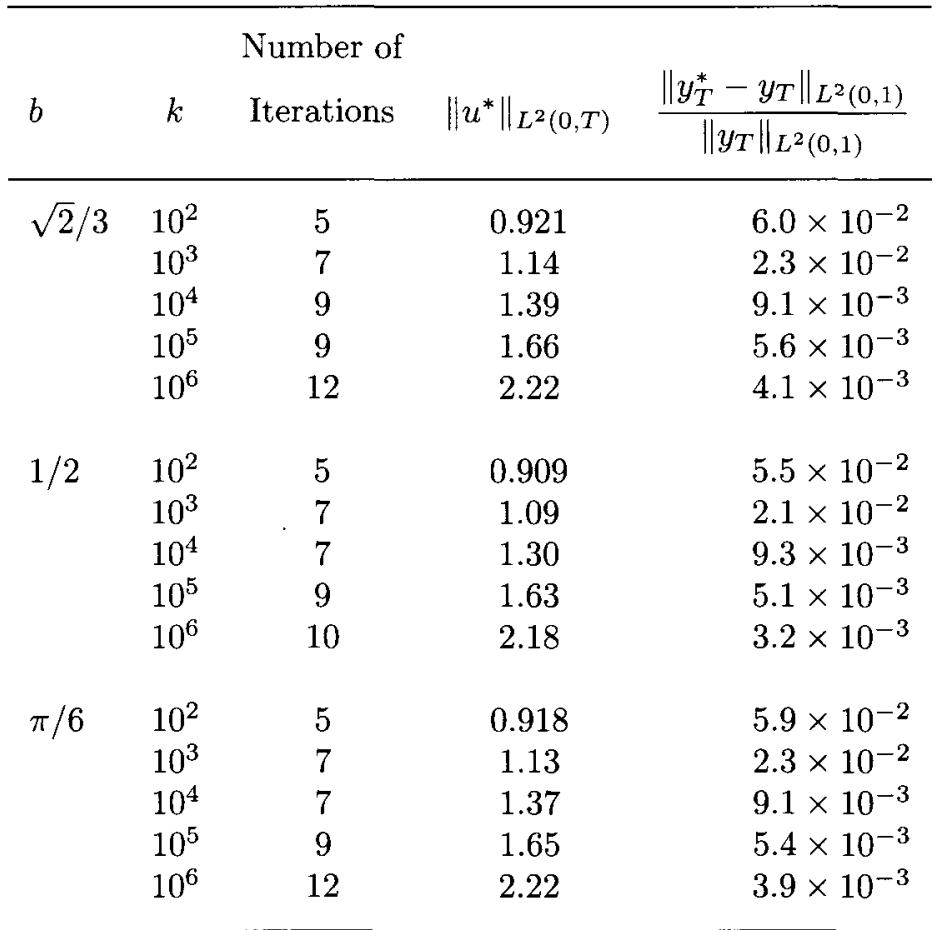


Table 2. Summary of numerical results (target defined by (1.462)); $T=3$, $h=\Delta t=10^{-2}$.

\begin{tabular}{|c|c|c|c|c|}
\hline$b$ & $k$ & $\begin{array}{l}\text { Number of } \\
\text { Iterations }\end{array}$ & $\left\|u^{*}\right\|_{L^{2}(0, T)}$ & $\frac{\left\|y_{T}^{*}-y_{T}\right\|_{L^{2}(0,1)}}{\left\|y_{T}\right\|_{L^{2}(0,1)}}$ \\
\hline \multirow[t]{6}{*}{$\sqrt{2} / 3$} & $10^{2}$ & 6 & 1.23 & $2.2 \times 10^{-1}$ \\
\hline & $10^{3}$ & 7 & 1.96 & $1.9 \times 10^{-1}$ \\
\hline & $10^{4}$ & 10 & 5.54 & $1.6 \times 10^{-1}$ \\
\hline & $10^{5}$ & 11 & 11.2 & $1.4 \times 10^{-1}$ \\
\hline & $10^{6}$ & 13 & 37.1 & $1.3 \times 10^{-1}$ \\
\hline & $10^{10}$ & 21 & 585 & $6.7 \times 10^{-2}$ \\
\hline \multirow[t]{6}{*}{$1 / 2$} & $10^{2}$ & 5 & 1.27 & $1.1 \times 10^{-1}$ \\
\hline & $10^{3}$ & 7 & 1.74 & $6.3 \times 10^{-2}$ \\
\hline & $10^{4}$ & 7 & 2.42 & $5.2 \times 10^{-2}$ \\
\hline & $10^{5}$ & 9 & 6.26 & $3.8 \times 10^{-2}$ \\
\hline & $10^{6}$ & 9 & 12.7 & $3.0 \times 10^{-2}$ \\
\hline & $10^{10}$ & 14 & 66.7 & $2.5 \times 10^{-2}$ \\
\hline \multirow[t]{6}{*}{$\pi / 6$} & $10^{2}$ & 5 & 1.24 & $1.9 \times 10^{-1}$ \\
\hline & $10^{3}$ & 7 & 1.84 & $1.6 \times 10^{-1}$ \\
\hline & $10^{4}$ & 9 & 4.84 & $1.4 \times 10^{-1}$ \\
\hline & $10^{5}$ & 12 & 9.87 & $1.2 \times 10^{-1}$ \\
\hline & $10^{6}$ & 15 & 29.7 & $1.1 \times 10^{-1}$ \\
\hline & $10^{10}$ & 21 & 534 & $5.9 \times 10^{-2}$ \\
\hline
\end{tabular}

solution of the discrete control problem. However, comment (iii) may apply to situations where the discrete control problem is really badly conditioned. The figures corresponding to the new results will be reported elsewhere. For $k=10^{2}, 10^{3}$ they are practically identical to the corresponding ones in Part I; for larger values of $k$ we still have a good agreement.

(2) In Section 1.10.7.3 of Part I, the target function $y_{T}(x)=27 x^{2}(1-x)$ was used for the numerical calculations, instead of the function $y_{T}$ defined by $(1.466)$, i.e. $y_{T}(x)=\frac{27}{4} x^{2}(1-x)$.

Table 4 shows the results obtained for $y_{T}(x)=\frac{27}{4} x^{2}(1-x)$ with the corrected conjugate gradient algorithm; the convergence is quite fast even for large values of $k$. 
Table 3. Summary of numerical results (target defined by (1.463)); $T=3$, $h=\Delta t=10^{-2}$.

\begin{tabular}{|c|c|c|c|c|}
\hline$b$ & $k$ & $\begin{array}{l}\text { Number of } \\
\text { Iterations }\end{array}$ & $\left\|u^{*}\right\|_{L^{2}(0, T)}$ & $\frac{\left\|y_{T}^{*}-y_{T}\right\|_{L^{2}(0,1)}}{\left\|y_{T}\right\|_{L^{2}(0,1)}}$ \\
\hline \multirow[t]{6}{*}{$\sqrt{2} / 3$} & $10^{2}$ & 5 & 0.92 & $3.47 \times 10^{-1}$ \\
\hline & $10^{3}$ & 8 & 2.3 & $3.11 \times 10^{-1}$ \\
\hline & $10^{4}$ & 10 & 7.3 & $2.72 \times 10^{-1}$ \\
\hline & $10^{5}$ & 11 & 16 & $2.46 \times 10^{-1}$ \\
\hline & $10^{6}$ & 13 & 26 & $2.4 \times 10^{-1}$ \\
\hline & $10^{10}$ & 28 & 2200 & $2.27 \times 10^{-1}$ \\
\hline \multirow[t]{6}{*}{$1 / 2$} & $10^{2}$ & 5 & 0.99 & $3.32 \times 10^{-1}$ \\
\hline & $10^{3}$ & 7 & 2.08 & $2.47 \times 10^{-1}$ \\
\hline & $10^{4}$ & 9 & 6.3 & $2.36 \times 10^{-1}$ \\
\hline & $10^{5}$ & 9 & 10 & $2.27 \times 10^{-1}$ \\
\hline & $10^{6}$ & 12 & 36 & $2.16 \times 10^{-1}$ \\
\hline & $10^{10}$ & 15 & 540 & $1.82 \times 10^{-1}$ \\
\hline \multirow[t]{6}{*}{$\pi / 6$} & $10^{2}$ & 5 & 0.94 & $3.43 \times 10^{-1}$ \\
\hline & $10^{3}$ & 7 & 2.4 & $3.02 \times 10^{-1}$ \\
\hline & $10^{4}$ & 9 & 6.9 & $2.66 \times 10^{-1}$ \\
\hline & $10^{5}$ & 10 & 16 & $2.40 \times 10^{-1}$ \\
\hline & $10^{6}$ & 13 & 29 & $2.34 \times 10^{-1}$ \\
\hline & $10^{10}$ & 28 & 2200 & $2.17 \times 10^{-1}$ \\
\hline
\end{tabular}


Table 4. Summary of numerical results (target defined by (1.466)); $T=3$, $h=\Delta t=10^{-2}$.

\begin{tabular}{lcccc}
\hline \multicolumn{5}{c}{ Number of } \\
$b$ & $k$ & Iterations & $\left\|u^{*}\right\|_{L^{2}(0, T)}$ & $\frac{\left\|y_{T}^{*}-y_{T}\right\|_{L^{2}(0,1)}}{\left\|y_{T}\right\|_{L^{2}(0,1)}}$ \\
\hline \multirow{2}{*}{$\sqrt{2} / 3$} & $10^{4}$ & 9 & 10.6 & $1.5 \times 10^{-1}$ \\
& $10^{5}$ & 12 & 18.4 & $4.0 \times 10^{-2}$ \\
& $10^{6}$ & 15 & 21.6 & $1.5 \times 10^{-2}$ \\
& $10^{10}$ & 16 & 34.0 & $8.3 \times 10^{-3}$ \\
$1 / 2$ & $10^{4}$ & 7 & 1.10 & $3.5 \times 10^{-1}$ \\
& $10^{5}$ & 9 & 1.38 & $3.5 \times 10^{-1}$ \\
& $10^{6}$ & 9 & 1.92 & $3.5 \times 10^{-1}$ \\
& $10^{10}$ & 9 & 3.28 & $3.5 \times 10^{-1}$ \\
$\pi / 6$ & $10^{4}$ & 6 & 10.1 & $1.8 \times 10^{-1}$ \\
& $10^{5}$ & 11 & 21.1 & $5.6 \times 10^{-2}$ \\
& $10^{6}$ & 12 & 26.7 & $2.1 \times 10^{-2}$ \\
& $10^{10}$ & 15 & 49.7 & $1.0 \times 10^{-2}$ \\
\hline
\end{tabular}


Table 5. Summary of numerical results (target defined by (1.467)); $T=3$, $h=\Delta t=10^{-2}$.

\begin{tabular}{|c|c|c|c|c|}
\hline$b$ & $k$ & $\begin{array}{l}\text { Number of } \\
\text { Iterations }\end{array}$ & $\left\|u^{*}\right\|_{L^{2}(0, T)}$ & $\frac{\left\|y_{T}^{*}-y_{T}\right\|_{L^{2}(0,1)}}{\left\|y_{T}\right\|_{L^{2}(0,1)}}$ \\
\hline \multirow[t]{5}{*}{$\sqrt{2} / 3$} & $10^{3}$ & 8 & 6.53 & $5.2 \times 10^{-1}$ \\
\hline & $10^{4}$ & 11 & 21.8 & $3.0 \times 10^{-1}$ \\
\hline & $10^{5}$ & 12 & 41.6 & $1.6 \times 10^{-1}$ \\
\hline & $10^{6}$ & 16 & 58.8 & $1.3 \times 10^{-1}$ \\
\hline & $10^{10}$ & 31 & 1900 & $7.1 \times 10^{-2}$ \\
\hline \multirow[t]{5}{*}{$1 / 2$} & $10^{3}$ & 7 & 2.05 & $7.1 \times 10^{-1}$ \\
\hline & $10^{4}$ & 9 & 2.68 & $7.1 \times 10^{-1}$ \\
\hline & $10^{5}$ & 9 & 6.27 & $7.1 \times 10^{-1}$ \\
\hline & $10^{6}$ & 9 & 12.6 & $7.1 \times 10^{-1}$ \\
\hline & $10^{10}$ & 15 & 66.7 & $7.1 \times 10^{-1}$ \\
\hline \multirow[t]{5}{*}{$\pi / 6$} & $10^{3}$ & 8 & 5.99 & $6.7 \times 10^{-1}$ \\
\hline & $10^{4}$ & 10 & 27.5 & $4.2 \times 10^{-1}$ \\
\hline & $10^{5}$ & 12 & 57.6 & $2.0 \times 10^{-1}$ \\
\hline & $10^{6}$ & 16 & 89.9 & $1.3 \times 10^{-1}$ \\
\hline & $10^{10}$ & 16 & 326 & $6.2 \times 10^{-2}$ \\
\hline
\end{tabular}

